Short Communication

\title{
Targeting Leishmania amazonensis amastigotes through macrophage internalisation of a hydroxymethylnitrofurazone nanostructured polymeric system
}

\author{
Lis Marie Monteiro a , Raimar Löbenberg ${ }^{b}$, Elizabeth Igne Ferreira a , Paulo Cesar Cotrim ${ }^{\text {, }}$ \\ Edite Kanashiro ${ }^{c}$, Mussya Rocha ${ }^{\mathrm{c}}$, Man Chin Chung ${ }^{\mathrm{d}}$, Nadia Bou-Chacra ${ }^{\mathrm{a}, *}$ \\ a Pharmacy Department, Faculty of Pharmaceutical Sciences, University de São Paulo, São Paulo, Brazil \\ ${ }^{\mathrm{b}}$ Faculty of Pharmacy and Pharmaceutical Sciences, University of Alberta, Edmonton, Canada \\ c Seroepidemiology, Cellular and Molecular Immunology Laboratory of the Institute of Tropical Medicine, University of São Paulo, São Paulo, Brazil \\ d Faculty of Pharmaceutical Sciences, UNESP-Araraquara, São Paulo, Brazil
}

\section{A R T I C L E I I N F O}

\section{Article history:}

Received 2 June 2016

Accepted 28 January 2017

\section{Keywords:}

Hydroxymethylnitrofurazone

Poly (n-butyl cyanoacrylate)

Leishmaniasis

Drug delivery

Polymeric nanoparticles

Dextran

\begin{abstract}
A B S T R A C T
Dextran-coated poly (n-butyl cyanoacrylate) nanoparticles (PBCA-NPs) were prepared and were evaluated for enhanced delivery of a promising anti-Leishmania drug candidate, hydroxymethylnitrofurazone (NFOH), to phagocytic cells. Currently available chemotherapy for leishmaniasis, such as pentavalent antimonials, presents low safety and efficacy. Furthermore, widespread drug resistance in leishmaniasis is rapidly emerging. To overcome these drawbacks, the use of nanosized delivery systems can reduce systemic drug toxicity and increase the drug concentration in infected macrophages, therefore improving treatment of leishmaniasis. PBCA-NPs containing NFOH (PBCA-NFOH-NPs) were prepared by an anionic emulsion polymerisation method. The z-average and polydispersity index (PDI) were determined by photon correlation spectroscopy, the zeta potential by microelectrophoresis and the entrapment efficiency by HPLC. Cytotoxicity was determined using macrophages from BALB/c mice. Efficacy tests were performed using Leishmania amazonensis promastigotes and amastigotes. The $z$-average of PBCA-NFOHNPs was $151.5 \pm 61.97 \mathrm{~nm}$, with a PDI of $0.104 \pm 0.01$, a zeta potential of $-10.1 \pm 6.49 \mathrm{mV}$ and an entrapment efficiency of $64.47 \pm 0.43 \%$. Efficacy in amastigotes revealed $\mathrm{IC}_{50}$ values of $0.33 \mu \mathrm{M}$ and $31.2 \mu \mathrm{M}$ for the nanostructured and free NFOH, respectively (95-fold increase). The cytotoxicity study indicated low toxicity of the PBCA-NFOH-NPs to macrophages. The selectivity index was 370.6, which is 49-fold higher than free NFOH (7.6). Such findings indicated that improved efficacy could be due to NP internalisation following site-specific drug delivery and reactivation of immune protective reactions by the NP components. Thus, PBCA-NFOH-NPs have the potential to significantly improve the treatment of leishmaniasis, with reduced systemic side effects.
\end{abstract}

(c) 2017 Elsevier B.V. and International Society of Chemotherapy. All rights reserved.

\section{Introduction}

The leishmaniases are diseases caused by protozoan parasites of the genus Leishmania, affecting approximately 12 million people worldwide. Several factors impair the successful treatment of leishmaniasis, the most notable being toxicity and parasite resistance [1]. Therefore, the search for new drugs and new delivery systems to overcome these issues are urgently needed.

Hydroxymethylnitrofurazone (NFOH) is a promising drug candidate that showed significant activity against Trypanosoma cruzi [2]. Its efficacy was evaluated and confirmed in a murine model of Chagas

\footnotetext{
* Corresponding author. Pharmacy Department, Faculty of Pharmaceutical Sciences, University de São Paulo, São Paulo, Brazil. Fax: +55 1130913621.

E-mail address: chacra@usp.br (N. Bou-Chacra).
}

disease [2]. Inhibition of trypanothione reductase by NFOH is the main mechanism of action. The reduced form of this enzyme is key for the redox defence system of trypanosomatids (including Leishmania) against oxidative stress in infected cells [3].

The ability of Leishmania parasites to survive and multiply within phagocytic cells from the skin, liver, spleen and bone marrow is a major factor and target for developing new active compounds and drug delivery systems against leishmaniases. The use of drug carriers, such as nanoparticles (NPs), can improve the therapeutic efficacy by releasing leishmanicidal compounds at the site of drug action [4], given the fact that NPs are preferentially taken up by macrophages following systemic or local administration. Consequently, this mechanism would be useful to increase treatment efficacy for leishmaniasis [5].

Among promising polymers used for NP preparation, poly n-butyl cyanoacrylate (PBCA) has gained increasing interest in drug delivery 
for pharmaceutical and medical applications owing to its biodegradability, low toxicity, ability to modify drug biodistribution, and ease of synthesis and purification [6].

One of the most common surfactants for PBCA-NP preparation is polymeric dextran. In addition to its interfacial properties, it has been described as a moiety to mannan and SIGN lectin receptors $[7,8]$. These receptors on macrophages recognise polysaccharides such as dextran, mannan and lipopolysaccharide from Gram-negative bacteria, allowing pathogen recognition, uptake and induction of immune responses. Taking advantage of this process, dextrancoated NPs have an excellent potential to increase intracellular drug concentrations and to reactivate immune responses that are disabled by Leishmania during mammalian infection [9].

In this work, we present the preparation and physicochemical characterisation of PBCA-NPs containing NFOH (PBCA-NFOHNPs), proposed as a means of active targeted drug delivery to macrophages, and its improved in vitro leishmanicidal activity against Leishmania amazonensis, with highly biocompatible characteristics.

\section{Materials and methods}

\subsection{Materials}

The n-butyl cyanoacrylate monomer was purchased from Braun (Melsungen, Germany). Dextran 70000, M199 culture medium and MTT reagent [3-(4,5-dimethylthiazol-2-yl)-2,5-diphenyl tetrazolium bromide] were all from Sigma-Aldrich (St Louis, MO). RPMI 1640 culture medium was purchased from Merck Millipore (Darmstadt, Germany). NFOH was synthesised by LAPEN-University of São Paulo (São Paulo, Brazil). All other reagents were of analytical grade.

\subsection{Preparation of n-butyl cyanoacrylate nanoparticles loaded with hydroxymethylnitrofurazone (PBCA-NFOH-NPS)}

PBCA-NPs were prepared by an anionic emulsion polymerisation method as described previously [10]. Briefly, dextran (1\% m/v) was dissolved in $0.1 \mathrm{~N} \mathrm{HCl}$ and the $\mathrm{NFOH}$ was dispersed. The monomer was then slowly added to the aqueous phase at $600 \mathrm{rpm}$ using a magnetic stirrer. After $4 \mathrm{~h}$ of polymerisation, the system was neutralised to $\mathrm{pH} 7.0$ using $0.1 \mathrm{~N} \mathrm{NaOH}$ to terminate the polymerisation process.

\subsection{Particle size distribution and zeta potential analysis}

The $z$-average and polydispersity index (PDI) were determined by photon correlation spectroscopy and the zeta potential was determined by microelectrophoresis using a Malvern Zetasizer Nano $\mathrm{ZS}^{\mathrm{TM}}$ (Malvern Instruments Ltd., Malvern, UK). Measurements $(n=3)$ were performed on NPs at $0.1 \%(\mathrm{v} / \mathrm{v})$ diluted in purified water adjusted to $50 \mu \mathrm{S} / \mathrm{cm}$ using $0.9 \% \mathrm{NaCl}$ solution.

\subsection{Transmission electron microscopy (TEM)}

TEM images of the NPs were taken using a JEOL $1010100 \mathrm{kV}$ microscope (JEOL Ltd., Tokyo, Japan) with the samples mounted on a copper-carbon grid and allowed to dry at room temperature.

\subsection{Determination of hydroxymethylnitrofurazone (NFOH) entrapment efficiency}

Regarding of the entrapped NFOH, $2 \mathrm{~mL}$ of freshly prepared dispersion was centrifuged for $30 \mathrm{~min}$ at $20000 \times \mathrm{g}$ at $25^{\circ} \mathrm{C}$. The supernatant containing the free drug was withdrawn and was analysed by high-performance liquid chromatography (HPLC). Drug loading was determined by the difference of the total amount of the NFOH minus the amount found in the supernatant. HPLC quantification was performed as previously described by Monteiro et al. [11].

\subsection{Efficacy assay against Leishmania amazonensis promastigotes}

Promastigotes $\left(1 \times 10^{6}\right)$ in early log phase were incubated in the presence of free NFOH, NP-bound NFOH and blank NPs $(4-12 \mu \mathrm{M})$ for $72 \mathrm{~h}$ at $24{ }^{\circ} \mathrm{C}$ with $5 \% \mathrm{CO}_{2}$ in M199 medium. Then, $20 \mu \mathrm{L}$ of MTT solution $(5 \mathrm{mg} / \mathrm{mL}$ ) was added to each well and was incubated for $4 \mathrm{~h}$. Sodium lauryl sulfate $(20 \% \mathrm{w} / \mathrm{v})$ was added to dissolve the formazan crystals and to obtain a homogeneous solution suitable for measurement of the absorbance at $595 \mathrm{~nm}$ using an enzymelinked immunosorbent assay (ELISA) microplate reader (Model 550 Microplate Reader; Bio-Rad, Hercules, CA). Viability was defined as the ratio (expressed as a percentage) of absorbance of treated cells to untreated cells. Experiments were performed in replicates of five and were repeated twice independently.

\subsection{Cytotoxicity}

In vitro tests were approved by the Animal Care and Use Committee of the Institute of Tropical Medicine of the University of São Paulo (São Paulo, Brazil). For evaluation of cytotoxic effects, murine peritoneal macrophages were extracted from mice via peritoneal lavage and were incubated in the presence of free NFOH, NPbound NFOH and blank NPs $(5-80 \mu \mathrm{M})$ with RMPI 1640 medium at $37{ }^{\circ} \mathrm{C}$ with $5 \% \mathrm{CO}_{2}$ for $24 \mathrm{~h}$. MTT solution $(5 \mathrm{mg} / \mathrm{mL}$ ) was added to each well and was incubated for $4 \mathrm{~h}$ and then sodium lauryl sulfate $(20 \% \mathrm{w} / \mathrm{v})$ was added. Absorbance was determined at $595 \mathrm{~nm}$. Experiments were performed in replicates of five and were repeated twice independently.

\subsection{Efficacy assay against Leishmania amazonensis amastigotes}

The amastigote assay was performed using peritoneal macrophages. The exudate was diluted with RMPI and was stained with trypan blue. Thereafter, macrophages $\left(2 \times 10^{5}\right)$ were seeded in 24-well plates and were incubated at $37^{\circ} \mathrm{C}$ with $5 \% \mathrm{CO}_{2}$ for $24 \mathrm{~h}$. Adhered macrophages were infected with promastigotes at a ratio of $5: 1$ and were incubated for $4 \mathrm{~h}$ at $34{ }^{\circ} \mathrm{C}$ and $5 \% \mathrm{CO}_{2}$. Extracellular parasites were then removed by washing with RPMI. Infected macrophages were further incubated in the presence of free NFOH, NP-bound NFOH and blank NPs (3-15 $\mu \mathrm{M})$ for $24 \mathrm{~h}$ at $37{ }^{\circ} \mathrm{C}$ in $5 \% \mathrm{CO}_{2}$. Finally, macrophages were washed with RPMI medium, were fixed in methanol, were stained using Giemsa and the amastigotes were counted (200 macrophages per replicate) using an optical microscope (Nikon, Tokyo, Japan). The selectivity was calculated as described by Benedict et al. [12]. Experiments were performed in replicates of five and were repeated twice independently.

\subsection{Determination of $I C_{50}$ and $C C_{50}$ values}

The concentration required to eliminate one-half of the parasites $\left(\mathrm{IC}_{50}\right)$ and the $50 \%$ cytotoxic concentration $\left(\mathrm{CC}_{50}\right)$ were expressed as the mean \pm standard deviation of parasite/cell viability. The $\mathrm{IC}_{50}$ was calculated by linear regression and the $\mathrm{CC}_{50}$ by non-linear regression analysis using GraphPad Prism v.5.01 (GraphPad Software Inc., La Jolla, CA). 


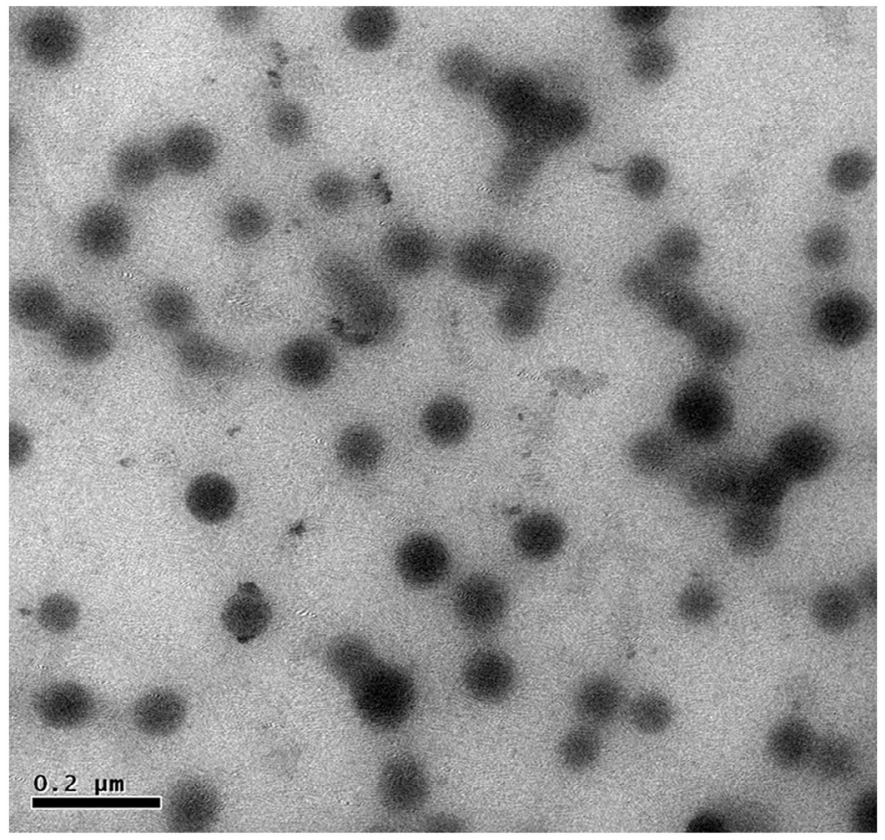

Fig. 1. Morphology of the poly (n-butyl cyanoacrylate) nanoparticles containing hydroxymethylnitrofurazone (PBCA-NFOH-NPs) by transmission electron microscopy showing the round shape and size of ca. $150 \mathrm{~nm}$, confirming the values from photon correlation spectroscopy. Magnification of $100 \mathrm{k} \times$. A high-resolution version of this slide for use with the Virtual Microscope is available as eSlide: VM03614.

\subsection{Statistical analysis}

The significance of differences among values was determined by analysis of variance (ANOVA) using Minitab 16 statistical software (Minitab Inc., State College, PA). A $P$-value of $<0.05$ was considered statistically significant.

\section{Results}

3.1. Hydroxymethylnitrofurazone-loaded poly (n-butyl cyanoacrylate) nanoparticles (PBCA-NFOH-NPS): physicochemical characterisation

The process yielded PBCA-NFOH-NPs of $151.5 \pm 61.97 \mathrm{~nm}$, with a PDI of $0.104 \pm 0.01$ (monomodal distribution) and a zeta potential of $-10.1 \pm 6.49 \mathrm{mV}$. The entrapment efficiency was $64.47 \pm 0.43 \%$. TEM showed well-rounded NPs (Fig. 1).

\subsection{Efficacy assay against Leishmania amazonensis promastigotes}

The NFOH-loaded NPs were significantly $(P \leq 0.05)$ more effective against promastigotes of $L$. amazonensis, with an $\mathrm{IC}_{50}$ value for NFOH-PBCA-NPs of $2.18 \mu \mathrm{M}$ compared with values of $19.04 \mu \mathrm{M}$ for free $\mathrm{NFOH}$ and $13.32 \mu \mathrm{M}$ for unloaded NPs.

\subsection{Cytotoxicity assay}

Cytotoxicity indicated that all treatments had low toxicity on uninfected macrophages. The $\mathrm{CC}_{50}$ was $122.3 \mu \mathrm{M}$ for NFOH-PBCANPs, $142.9 \mu \mathrm{M}$ for unloaded NPs and $237.0 \mu \mathrm{M}$ for free NFOH.

\subsection{Efficacy assay against Leishmania amazonensis amastigotes}

The leishmanicidal activity of the NFOH-loaded NPs was significantly $(P<0.05)$ increased $\left(\mathrm{IC}_{50}=0.33 \mu \mathrm{M}\right)$. The $\mathrm{IC}_{50}$ was $31.2 \mu \mathrm{M}$ for the free drug and $25.1 \mu \mathrm{M}$ for blank NPs (Fig. 2). The selectivity indexes were 370.6 for NFOH-PBCA-NPs and 7.6 for the free drug.

\section{Discussion}

The improved activity of drug-loaded NPs presented here highlights the relevance of this class of delivery system for leishmaniases.

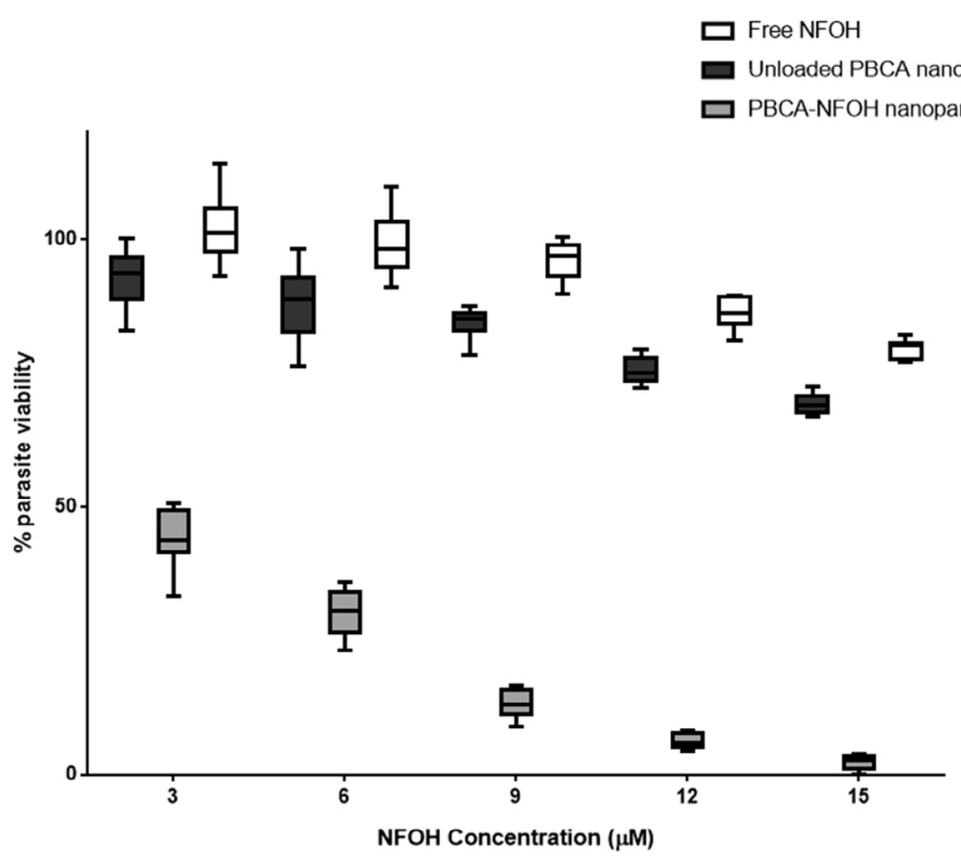

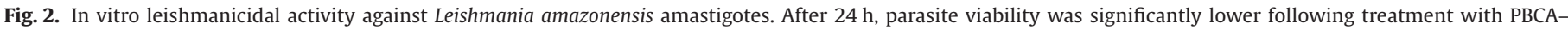

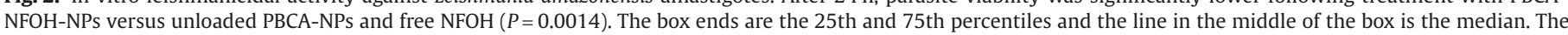

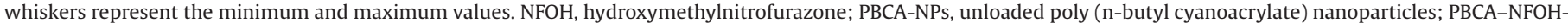

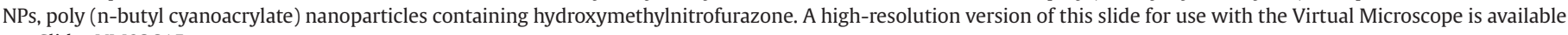
as eSlide: VM03615. 


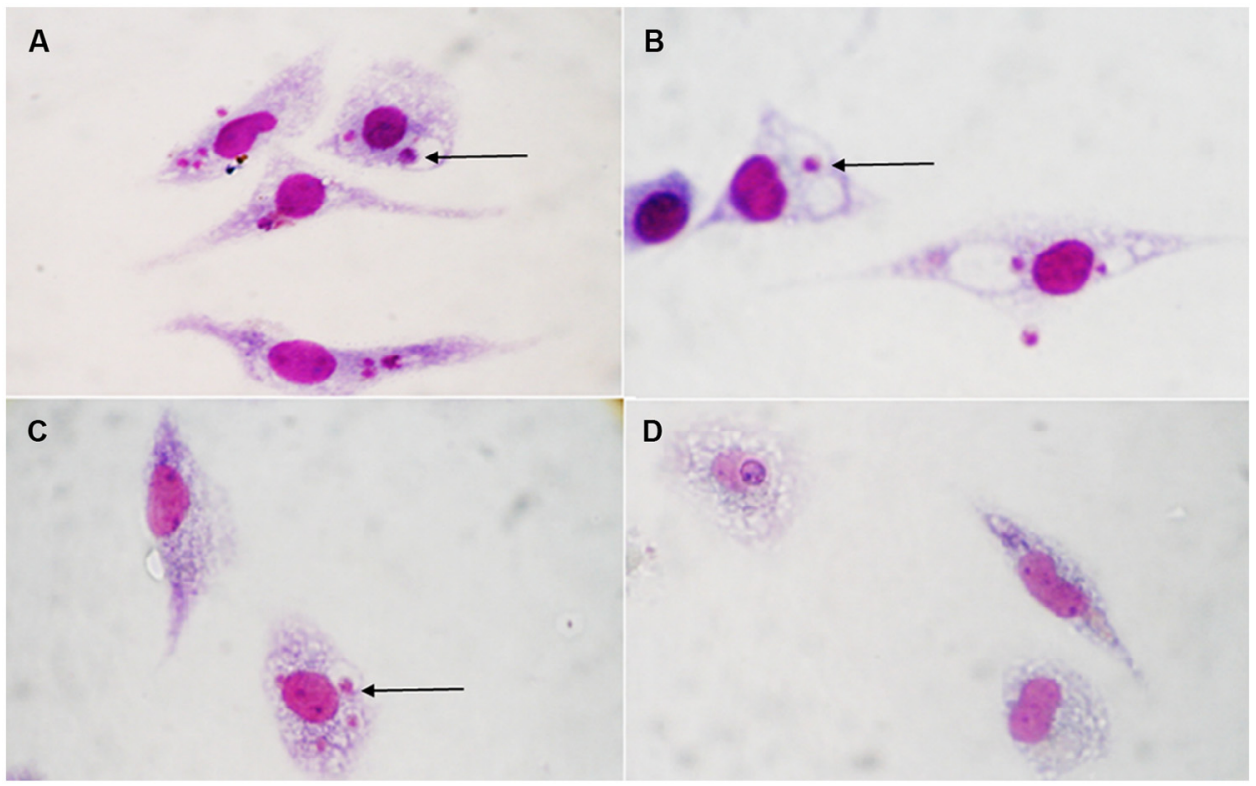

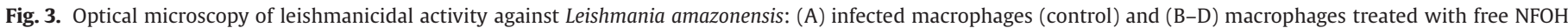

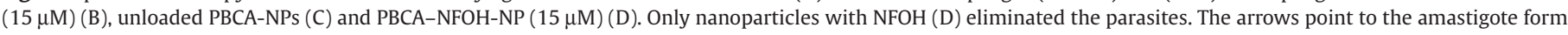

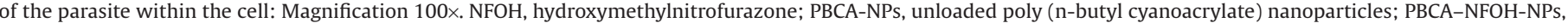
poly (n-butyl cyanoacrylate) nanoparticles containing hydroxymethylnitrofurazone.

The PBCA-NFOH-NPs $z$-average ( $151.5 \pm 61.97 \mathrm{~nm}$ ) was similar to those obtained by Weyermann et al. and Sommerfeld et al. [13,14], who reported a $z$-average equal to $130-140 \mathrm{~nm}$ and $164-326 \mathrm{~nm}$, respectively. The size of the NP determines its targeting abilities such as cell uptake. The particles used in this study were within the optimal size range for particles to be taken up by phagocytosis $[15,16]$.

Smaller particles $(<100 \mathrm{~nm})$ are preferably internalised by endocytosis, which occurs in virtually all cells and is essential for nutrient uptake and intracellular communication. Thus, PBCA-NFOH-NPs are promising drug delivery systems to target macrophages. Taking into account that dextran, a polysaccharide widely used to prepare PBCA-NPs, sterically stabilises NPs, the zeta potential value of $-10.1 \mathrm{mV}$ can be considered as suitable to stabilise the NPs in suspension. Stability was confirmed (data not shown) for $\geq 3$ months in $20 \mathrm{~mL}$ borosilicate vials at $8 \pm 2{ }^{\circ} \mathrm{C}$.

Regarding the activity tests for the promastigote form of the parasite, the mean $\mathrm{IC}_{50}(2.18 \mu \mathrm{M})$ of PBCA-NFOH-NPs was 8.7-fold lower than of free NFOH $(19.04 \mu \mathrm{M})$ and 6.1-fold lower than the unloaded NPs $(13.32 \mu \mathrm{M})$. The cytotoxicity $\left(\mathrm{CC}_{50}\right)$ of free $\mathrm{NFOH}$ $(237.0 \mu \mathrm{M})$ and PBCA-NFOH-NPs $(122.3 \mu \mathrm{M})$ allowed the activity assay using the amastigote form of the parasite. This assay revealed that the antileishmanial activity was due to the PBCA-NFOHNPs. The cytotoxicity assay showed that non-infected macrophage functions were not impaired.

The remarkable increase in the activity of the PBCA-NFOH-NPs revealed the potential application of this formulation for targeted delivery to macrophages. Optical microscopy (Fig. 3C,D) showed disseminated vacuoles in the cytoplasm when the cells were treated using NPs. This suggested their successful internalisation into macrophages. As a consequence, the $\mathrm{IC}_{50}$ of the NFOH-loaded NPs $(0.33 \mu \mathrm{M})$ was 95 -fold lower than that of free NFOH $(31.2 \mu \mathrm{M})$. Although the $\mathrm{CC}_{50}$ of NPs was higher for free NFOH, the PBCA-NFOHNP selectivity index (370.6) was 49-fold higher than the free drug (7.6), which showed potential use for improving leishmaniases treatment safety.

These findings indicated remarkable performance of PBCANPs, which could be due to the sustained release and protection of the drug from degradation. In addition, reactivation and induction of immune responses could play a major role, as explained by the work of Sarfraz et al. [17]. They showed that PBCA-NPs could cause an immunoreaction in macrophages, which can have a positive therapeutic effect against cancer cells. Similarly, the current results show that PBCA-NPs have a superior antileishmanial effect. Whether this is due to immunological changes in the macrophages owing to NP treatment as reported in the above study has to be further investigated.

The NPs described in this work, when incorporated into a topical formulation or injected into a wound, can be internalised by macrophages, which are recruited to the site during cutaneous leishmaniasis infection [18]. Likewise, NPs can be injected intravenously. Considering the fact that phagocytic cells are responsible for clearing and degrading any foreign matter from the bloodstream [19], these NPs can be rapidly internalised by cells from the liver and spleen, which are the most affected organs in visceral leishmaniasis [20]. In the above routes of administration, the NPs can increase the drug concentration at the site of action and avoid redistribution to the systemic circulation. Therefore, the in vitro efficacy found in the present work might be correlated with in vivo efficacy in a whole organism owing to these mechanisms.

\section{Conclusion}

In this work, NPs loaded with an antileishmanial drug candidate (NFOH) were successfully prepared with dextran as an actively targeted drug delivery system to macrophages. The PBCA-NFOHNPs showed potential increased efficacy and selectivity compared with free NFOH. These results can be explained by intracellular drug accumulation following NP internalisation by macrophages, assisted by their recognition by mannan and SIGN lectin receptors. Moreover, PBCA-NPs may stimulate immune responses in macrophages, since unloaded NPs also presented substantial antiLeishmania activity. As a result of these findings, the presented nanostructured system may be a promising and rational alternative 
for the treatment of leishmaniases and should be investigated further in in vivo and immunological studies.

\section{Acknowledgments}

The authors thank the United States Pharmacopeial Convention USP-Brazil for providing support for the high-performance liquid chromatography (HPLC) analyses. The paper was revised by Jim Hesson.

Funding: None.

Competing interests: None declared.

Ethical approval: In vitro tests were approved by the Animal Care and Use Committee of the Institute of Tropical Medicine of the University of São Paulo (São Paulo, Brazil) [no. CPE-ITM/000269A].

\section{References}

[1] Freitas-Junior LH, Chatelain E, Kim HA, Siqueira-Neto JL. Visceral leishmaniasis treatment: what do we have, what do we need and how to deliver it? Int J Parasitol Drugs Drug Resist 2012;2:11-19.

[2] Davies C, Marino Cardozo R, Sánchez Negrette O, Mora MC, Chung MC, Basombrío MA. Hydroxymethylnitrofurazone is active in a murine model of Chagas' disease. Antimicrob Agents Chemother 2010;54:3584-9.

[3] Dumas C, Ouellette M, Tovar J, Cunningham ML, Fairlamb AH, Tamar S, et al. Disruption of the trypanothione reductase gene of Leishmania decreases its ability to survive oxidative stress in macrophages. EMBO J 1997;16:2590-8.

[4] Costa Lima S, Rodrigues V, Garrido J, Borges F, Kong Thoo Lin P, Cordeiro da Silva A. In vitro evaluation of bisnaphthalimidopropyl derivatives loaded into pegylated nanoparticles against Leishmania infantum protozoa. Int J Antimicrob Agents 2012;39:424-30.

[5] Almouazen E, Bourgeois S, Boussaïd A, Valot P, Malleval C, Fessi H, et al. Development of a nanoparticle-based system for the delivery of retinoic acid into macrophages. Int J Pharm 2012;430:207-15.

[6] Duan J, Liu M, Zhang Y, Zhao J, Pan Y, Yang X. Folate-decorated chitosan/ doxorubicin poly(butyl)cyanoacrylate nanoparticles for tumor-targeted drug delivery. J Nanopart Res 2012;14:761.
[7] Kang YS, Yamazaki S, Iyoda T, Pack M, Bruening SA, Kim JY, et al. SIGN-R1, a novel C-type lectin expressed by marginal zone macrophages in spleen, mediates uptake of the polysaccharide dextran. Int Immunol 2003;15:17786.

[8] Choi R, Yang J, Choi J, Lim EK, Kim E, Suh JS, et al. Thiolated dextran-coated gold nanorods for photothermal ablation of inflammatory macrophages. Langmuir 2010;26:17520-7.

[9] Gupta G, Oghumu S, Satoskar AR. Mechanisms of immune evasion in leishmaniasis. Adv Appl Microbiol 2013;82:155-84.

[10] Sham JO, Zhang Y, Finlay WH, Roa WH, Löbenberg R. Formulation and characterization of spray-dried powders containing nanoparticles for aeroso delivery to the lung. Int J Pharm 2004;269:457-67.

[11] Monteiro LM, Tavares GD, Ferreira EI, Consiglieri VO, Bou-Chacra NA, Löbenberg R. Reverse phase high-performance liquid chromatography for quantification of hydroxymethylnitrofurazone in polymeric nanoparticles. Braz J Pharm Sci 2015;51:561-7.

[12] Benedict A, Bansal N, Senina S, Hooper I, Lundberg L, Fuente C, et al. Repurposing FDA-approved drugs as therapeutics to treat Rift Valley fever virus infection. Front Microbiol 2015:6:676.

[13] Weyermann J, Lochmann D, Georgens C, Rais I, Kreuter J, Karas M, et al. Physicochemical characterisation of cationic polybutylcyanoacrylatenanoparticles by fluorescence correlation spectroscopy. Eur J Pharm Biopharm 2004;58:25-35.

[14] Sommerfeld P, Schroeder U, Sabel BA. Long-term stability of PBCA nanoparticle suspensions suggests clinical usefulness. Int J Pharm 1997; 155:7.

[15] Lin Y, Pan Y, Shi Y, Huang X, Jia N, Jiang JY. Delivery of large molecules via poly(butyl cyanoacrylate) nanoparticles into the injured rat brain. Nanotechnology 2012;23:165101.

[16] Weiss CK, Lorenz MR, Landfester K, Mailänder V. Cellular uptake behavior of unfunctionalized and functionalized PBCA particles prepared in a miniemulsion. Macromol Biosci 2007; 7:883-96.

[17] Sarfraz M, Roa W, Bou-Chacra N, Löbenberg R. Inflammation caused by nanosized delivery systems: is there a benefit? Mol Pharm 2016:13:3270-8.

[18] Mahdavian DB, Van der Veer WM, van Egmond M, Niessen FB, Beelen RH. Macrophages in skin injury and repair. Immunobiology 2011;216:753-62.

[19] Walkey CD, Olsen JB, Guo H, Emili A, Chan WCW. Nanoparticle size and surface chemistry determine serum protein adsorption and macrophage uptake. J Am Chem Soc 2012;134:2139-47.

[20] Bhattacharjee A, Majumder S, Majumdar SB, Choudhuri SK, Roy S, Majumdar S. Co-administration of glycyrrhizic acid with the antileishmanial drug sodium antimony gluconate (SAG) cures SAG-resistant visceral leishmaniasis. Int J Antimicrob Agents 2015;45:268-77. 